\title{
Welcome to the jungle: science communication in the mediatized society
}

\author{
Tea Čonč, Denis Kos \\ Faculty of Humanities and Social Sciences, University of Zagreb \\ Ivana Lučića 3, 10000 Zagreb \\ \{tconc, dkos\}@ffzg.hr
}

\section{Summary}

This paper discusses the influence of the media on science communication, on both the institutional and personal level with regard to the changing public nature of science. Authors analyze three heavily intertwined parts of the modern system of science: regulation of science, communication of science and the processes of its production. Looking from the institutional perspective the following tendencies of science mediatization are observed: institutional and individual self-promotion (i. e. PR), increase of scientific (and/or political) impact, selfregulatory behaviour and simplistic mediation of scientific content. At the micro-level, or in the arena where science actually is produced three areas of particular media influence are highlighted: achieving and improving scientific status, increase of informal scientific communication and changes in scholarly publishing patterns. Authors conclude by emphasizing the need of embedding the media and information literacy content into all levels of science education and production.

Keywords: science, science communication, mediatization theory, media

\section{Introduction}

In this paper we explore the influence of the media on science communication. Today, science, scientists and scientific topics are much more exposed to the media than they were ever before. At the same time the overall social influence of the media is increasing. Stig Hjarvard, a media theoretician, observes that the media ,mould the way people communicate, act and sustain relationships with each other" [2]. This author points to a necessity of examining how science as an older activity is changing under the influence of the rather recent phenomenon (i.e. the media). This particular influence is the focus of study of a new media concept called mediatization, which has emerged in the last few years and which aims to research how different existing social systems change their inner rules and the ways they function to adopt (more or less consciously) a rationale based on the media logic. Although there have already appeared critical debates 
on the subject and the method of mediatization theory ${ }^{1}$, we attempt a review of articles on the topic of mediatization of science ${ }^{2}$. To achieve this, we combine insights from the field of information and communication sciences that can shed valuable light on this topic of interest.

There are two main approaches in studying the processes of mediatization, and this paper adopts both of them, each with regard to the theoretical background it originates from. First is the institutionalist approach where mediatization is seen as a process in which non-media social actors have to adapt to media's rules, aims, production logics [2] but also its constraints - an approach that originates from the discourse of the traditional mass media and the "broadcast era" [4]. The second approach looks at media from a socio-technical standpoint which sees mediatization as a process in which the changing information and communication technologies drive the changing communicative construction of culture and society [2] and which is usually connected to the uprise of the new media. In the end, we look how these two approaches affect the scientist himself who is charged with the responsibility of science production.

Underlying these approaches is a concrete definition of the media as a transformational pervasive instrument where the conflicts in different social systems are being reworked in a media space and time according to the principles of media logic. Such a definition constitutes the media as a growing colonizing force; a form through which phenomena are presented and which ultimately wants to become the (most) relevant point of the legitimation and construction of the social order, consequently reshaping other social subsystems according to its logic. This outlook has been called the "centrality of the media" [1] and will be considered as a point of reference in this paper.

Still, we look at science and scientific communication, as one of the older and most prominent of social systems, in context of a society that is becoming ever more globalized, technological, economized but also, as the theory argues mediatized. Not putting aside the common problems of mediatization theory we will attempt to engage the problem of a mediatized science by taking into account the role of the non-media factors such as cultural, political and social variables considering at the same time relevant aspects at the macro-, meso- and the micro- level, as proposed by Deacon and Stanyer [2] . Moreover, our main focus will not be the media and the mediatic turn themselves, so much as the processes that embody the contemporary practice of science. The key question

\footnotetext{
${ }^{1}$ For the insight in the most recent debate see [2], [3].

${ }^{2}$ For a concise introduction into the mediatization theory see the short overviews in Hepp, Andreas. Cultures of mediatization. Cambrigde: Polity Press, 2013, and Hjarvard, Stig. The mediatization of culture and society. Park Square: Routlege, 2013. Politics were the first research field of this uprising theory, because of the easily perceived connections it has with the media. Strömback proposes four phases of mediatization of politics which imply a long-term process but which do not necessarily have to coincide with any specific time periods. [15]
} 
to be answered is how can science become mediatized and is it possible to pinpoint the exact processes affected? Science is a complex endeavour. It is impossible to examine all of its aspects since its functions, aims and purposes are much more varied than those of the media system. To find answers we look at science through a model consisting of three heavily intertwined moments or parts of the modern system of science which can encompass the institutionalist and the socio-technical approaches, namely: the regulation of science, communication of science and the processes of its production.

\section{Regulation of science and its changing public nature}

We regard the processes of regulation in science as the overarching structure that organizes and legitimizes scientific work in the society. It refers to the institutional and managerial arrangements that are formed through legal regulations and executive academic and governmental instances. From the viewpoint of communication theory, regulation of science is regarded as a conversation between relevant stakeholders. Due to the historically achieved autonomy of science this conversation has the quality of a debate. The way science is and can be done today is a result of a long history of an ongoing negotiation between the scientists, the general public and the state, and nowadays also the corporate sector. Since the 17th century science fought for a primacy in the value of the knowledge it produces and has enjoyed a privileged status. However, due to the quality of its relations with the aforementioned stakeholders this status has been challenged. This problem, dubbed in literature the changing public nature of science (e.g. [10]), is becoming complicated even further by the role that the media play in it.

To understand the problem of the public nature of science we have to look closer at the context in which science exists today. According to Nowotny [10] we can distinguish two main ways the public nature of science is changing. These two influences can be regarded as somewhat opposite to each other. On one hand, there is the proprietization of science - the produced data and achievements that are increasingly being regarded as something that is private ownership of the scientist or the institution that produced them - that "support[s] the transformation of public science into privately owned knowledge domains" [10]. On the other hand, there is a growing demand for access and public oversight of new achievements that are guided by a will "to preserve a sense of control over one's own life in a bewildering world of scientific-technological complexity, intertwined with the relentlessly ongoing process of globalization" [10]. Still, the inner workings of science, the rigour of its method and approach, remain the same. Furthermore, regardless of the current demands, either towards democratization or privatization, its task to systematically discover and present gained understandings about the universe did not change. 
At the first look one notices that, in the scope of the regulation of science, the problem of the public nature of science arises in the field of scientific communication, since the produced knowledge (that one can subsequently claim ownership of) has to be communicated and validated. At the second look, as Nowotny has also mentioned and still in the field of communication, the problem also exists in the shaken authority of science to communicate the Truth on Nature's behalf since the general public feels left out i.e. kept on the border of true understanding of contemporary scientific endeavours. Such a definition of the problem identifies two audiences [10]: the "insider" scientific and the "outsider" general public that interact with the newly produced tentative contributions to the ever-changing body of scientific knowledge.

These insights lead us to conclude that because science is increasingly adopting an economic logic [14], it has removed itself from the field of perceived public interest. Under the influence of proprietization and by adoption of economic criteria public science is changing its research priorities and presents itself as obliged to get public approval. On the contrary, as Marcinkowski and Kohring [9] argue: "given the new schemes of public funding, the public communication of science primarily serves the purpose of enabling academic institutions to promote themselves in a competition that has been forced upon them by the political domain" [9]. Science is only in appearance becoming increasingly accountable to the public. If we consider that proprietization needs a climate of deregulation, politics is seen as withdrawing "from its original responsibility of making binding decisions" [9] that configure a public accountability of science. Then, science itself becomes less focused on the need to be accountable to the public and more bound by the need to achieve efficiency. Following the insights gained from mediatization theory it is the media who have taken upon them to hold science accountable and make its inner struggles visible to the public, while they are also providing space and time to individual scientists and institutions for their much needed promotion.

\section{Communicating science to the public}

In this chapter we first deal with the "outsider" public of scientific communication. In the reviewed literature, communication to this public is looked through the lens of the field of public relations (PR) mediated mostly through the traditional mass media. To start off with some clarifications on what kind of PR we are at this point referring to, Marcinkowski and Kohring point out that we are not dealing with the question whether the public can play any significant role in deciding what counts as scientific knowledge, since the truthfulness of insights does not change if they are reviewed by the public. The mechanism for declaring something is scientific knowledge still predominantly is the peer-review process coupled with formal scientific criteria that communicated contributions need to integrate. Science is not a genuine public business [9] and its main audience is not the general public [14]. Even so, the pervasive impact of the media 
seen mainly from the institutionalist perspective reveals four notable tendencies of science mediatization:

- institutional and individual self-promotion,

- increase of scientific (and/or political) impact,

- self-regulatory behaviour and

- simplistic mediation of scientific content.

All of the various forms of public communication are increasingly entering into the media domain. Scientific institutions have reacted to this fact, just like many other institutions. They have adopted the non-scientific game of self-representation in order to publicly legitimize themselves and acquire socio-political relevance. As a result, science related media communications have increased, both within institutions and among individual scientists. [12] At the institutional level we can recognize that new public information (or PR) departments are being established in order to develop appropriate media strategies for public presentation of the institution and create and manage opportunities for scientists to appear in the media "as they preselect and produce stories anticipating the journalistic criteria"[11]. We have to note that the aim of these efforts is not to publicly present the latest scientific achievements, institution's ongoing projects or its future plans etc., rather their aim is to make sure that the institution "looks good" - that it has the possibility to manage and control its public image. Institutions can also "look good" by taking stands in public discussions and political debates or give opinions on emerging social crises (be those political, health or related to other issues). According to Peters, [12] these opinions will later be adopted and repeated by politicians who have the executive power to enable that the scientific expertise has the chance to become a relevant influence in policymaking. This inclusion might be a double-edged sword if politicians use this expertise for justification - to manage the appearance of their proposals, while the content and the estimation of value come in second. This relationship opens up a space for various other kinds of political pressures on science which recurrently stimulate a scientific institution to plan a so-called "crisis management strategy" to defend itself from journalists' attacks when, for example, financial or revision reports, or some incorrect or misleading information is published. To reiterate, science is distraught between the need to promote itself, defend its image, manage its influence and actually be publicly accountable, true to the principles of scientificity and efficient in economic terms without having the chance to rely on any (legally based) mechanisms of control. A further aspect of science mediatization is revealed in scientific institutions attempt to develop their own "media and communication strategy" that can ensure popularity and increase their influence. Peters observes that this media orientation originates from an understanding that the interaction with media is a part of the definition of a modern scientist's role, especially regarding his leadership capabilities. At the same time, institutions are trying to monitor and influence its scien- 
tists' media related activities, mostly by subtle means, to make sure they conform to the adopted media strategy. [11]

Mediatization of science spreads the criteria of media logic even between individual scientists. They observe the media interactions of their colleagues and evaluate how they established their competence as scientists in the media, the content of their statements, their self-presentation, the reputation of the news organization they appear at and the level of priority they give to a scientific way of speaking. [11] We can conclude that scientists estimate their colleagues' media interactions in order to learn from their experiences and regulate their own behaviour with regard to "what works" based on the goal of their media appearance.

Finally, the most familiar aspect of the mediatization of science probably refers to media presentations of scientific topics and the expression of journalistic views on science in the media. From the scientific point of view reporting on scientific topics is often too simplistic and exclusive in a combination with the dominant sensationalist approach (for the Croatian example see Šljok and Vuković Brajdić [16]). Research topics are chosen according to their current potential to entice attention [9] resulting in behaviour typical for the media [17]. Although traditional mass media still present the most commonly used way in which scientific institutions communicate their messages, the process of their recognition of the new media has already started. An example is given by Lövgren and Pallas where a university used blog posts by its vice-chancellor for public promotion that favour market and competition-oriented claims with a dominance of reputation, legitimacy and status based communication. [7] A similar example we find in the work of Lüthje who emphasised that most of the scientific blogs are in fact official PR blogs of scientific institutions. [8]

\section{Producing science in an embedded communication environment}

We have talked about how practices of scientific institutions change under the influence of mediatization. In this last chapter we focus on the individual scientist and move away from communication of science mediated through traditional mass media towards the entangled structure of democratized communication and science production through the new media. The new media establish an embedded communication environment where science production is immersed, soaked in a network created by a plethora of technological gadgets and applications which are not just tools, but also communicational devices that organize, facilitate and connect scientists and/or their contributions. ${ }^{3}$ Through the interactive new media like social networks, forums, blogging and microblogging services, collaborative content creation platforms such as wikis, etc. the border between internal and external communication of science is getting blurred.

\footnotetext{
${ }^{3}$ Herein we refer to research data, presentations, and textual scientific contributions.
} 
Since it is mediated through these network-based media science communication gains an aura of accessibility. Web 2.0 based technologies offer a decentralized structure where individual scientists or science enthusiasts (as members of the general public) can create a voice of their own and publish content about science. This process, called disintermediation, undermines centralized mediation, a trait found in the mass media system. Still, although there is no intermediary, the question remains whether Web 2.0 based media construct a logic of their own, and give way to a different, socio-technical mediatization of science.

At this micro-level, we noticed three aspects of the mediatization of science that have direct impact on its production:

- achieving and improving scientific status,

- increase of informal scientific communication, and

- changes in scholarly publishing patterns.

With the help of the new media, scientific institutions or individual scientists can completely avoid journalist mediation and thus "maintain control over the process and content of communication" [11]. Activities carried out online, may directly improve not only public but also the scientific status of the individual scientist. New research is needed that would correlate the scientific impact of individual scientists with the creation of their media presence on the web. On the other side, mediatization might not reveal itself only as an increase but also as a decrease in scientific impact. In this decentralized media space characterized by disintermediation questionable content can easily gain influence. To combat these influences in science the scientific community is using Web 2.0 based media as an approachable way to reveal pseudoscientific work, for example DC's improbable science blog [5]. Still, the truth is only in the eye of the beholder - ultimately, he will determine whether these accusations are grounded, or new media are used to undermine particular scientists or research agendas. It is in these cases that mediatization of science presents its influence to reconstruct science as an autonomous social system into a contested area for resources and primacy of outlooks.

The intensification of scientist's online activities leads to an overall increase in informal scientific communication. Public informal communication of science is also getting more and more popular, for example between scientists and the interested public or only among citizens. [8] Examples of informal communication between scientists, experts and the public can best be found on (micro)blogging platforms. Coupled with the fragmentation of communication, important topics ${ }^{4}$ are being discussed e.g. on Twitter. This kind of communication has accepted the boundaries posed by the media form. Sentences are short, one

\footnotetext{
${ }^{4}$ One such case can be found under \#acrlrevisions where a number of academics and practitioners discuss the new proposal to revise the American College and Research Libraries Association's Information literacy competency standards for higher education. These debates were later summarized on blogs and articles published in online journals in the field.
} 
has to use a lot of abbreviations to save space and thoughts are maximally compressed. Still, an estimation of the true influence of this kind of communication eludes us. Furthermore, these kinds of informal discussions appear to be used to establish what positions exist and what information is available. Before these particular opinions, interpretations and local knowledge gained from (e.g. professional) experience can sway the dominant position they could serve to establish dialogue in an extended peer community during public debate (about legal propositions, state-wide practical guidelines, strategy formation etc.). Interestingly enough, research shows that Web 2.0 tools are not widely used among scientists [6] and that science still resists the processes of mediatization. [13] It has also been shown that younger scientists are keener to use these tools. [6] [8] This leads us to question how will the dominant scientific communication and production processes look like when young scientists, some of them digital natives, become the leading generation. In this highly mediatized information jungle, besides the scientific skills and knowledge, scientists have to achieve a very high level of media and information literacy. Finally, the mediatized environment is undoubtedly changing patterns of scholarly publishing. Online social networks for scientists enable self-archiving of scientific contributions. Scientists can act on their own due to the possibilities offered by the media and communicate their contributions on their own terms backed by licensing management options like the Creative Commons licences. With the wide-spread of open access initiatives and the increasing number of platforms and tools more and more scientists are becoming aware of the benefit of publishing in open access. Decentralized new media stimulate the openness of science and create a space of influence to change the dominant publishing patterns in science. The appeal of such practices resides in the possibilities the technology offers, since it resonates with the principle of transparency of scientific work - science is given the chance by the new media to mediate its processes publicly from allowing open corroboration of results by posting online the raw research data, publishing pre- and post-print versions of scientific contributions, to the transparent peer-review process and the final act of publishing in open access. Multinational publishing corporations are facing important decisions whether to accept open access initiatives as relevant stakeholders. Rejecting any adjustments to their publishing policies and keeping their original positions could make them become obsolete, since open platforms like arXiv.org enable that scientists themselves maintain the control over the publishing process as a whole.

It is important that (future) scientists have all the tools, skills, knowledge and information needed for orientation in this new scientific (and social) environment. With respect to these changes a scientist's education at all levels has to integrate an emancipatory quality so he/she is able to distinguish the useful from invasive influences. The field of information literacy already debates these needs in context of Research 2.0. In a paper by Koltay, Špiranec and Karvalics [6] information literacy practices of academic libraries are put on the crossroads 
as support amidst the changes in the research process brought about by Web 2.0 on one hand and the information needs of individual researchers on the other. From the standpoint of the complex of science, media and information literacies, all involved in Research 2.0, we return to what we have emphasised in the beginning - to the individual researcher and his/her responsibility to uphold the principles of scientificity who understands how these principles are changing under the influence of mediatization and as an engaged and critical information literate preventively ensures that "good" publicly accountable science is done in the first place.

\section{Conclusion and further research recommendations}

In this paper we identified two pressure directions that act upon today's science. Above, from the institutional level there are political and economic pressures which make science distraught between the needs to publicly promote itself, defend its image and manage its influence and accountability. While struggling with these pressures in the mediatized environment (presented mainly through traditional, centralized mass media) the influence of the media logic is a potential threat to the autonomy and quality of scientific research. Further research could show whether criteria of scientific efficiency have succumbed to media logic and explore the policy makers' awareness of this influence. On the other hand, there is a growing demand for access and public oversight, but according to the dominant opinion among the theoreticians, public opinion is not indispensable for science credibility. Not to be misunderstood, we do not want to deny that science has to answer to society, and especially so if it is publicly funded, but we argue that media related PR activities might not be the adequate mechanism of science accountability. It should be regulated with mechanisms that are independent from the media influence before any kind of media representation takes place. Qualitative research of media content could elicit media involvement in science regulation. Also, with respect to the institutional communication of science, the foci of future research need to be the practices of institutions and individual scientists that show how they construct media and communication strategies in order to identify and promote best practices. The second part of the article looks at science mediatization processes from below adopting the socio-technical view that reveals how decentralised new media democratize communication and access to scientific information. These processes change the way scientists do science by strengthening informal science communication and by changing publishing practices. Still, a crucial area for further developments in the field needs to be the investigation of the impact of digital scientific waste that overflows the web amplifying the need to develop eco-friendly models of science production. Finally, we believe that to be prepared to deal with these changes it is of great importance that scientists have all the media and information literacy skills and knowledge needed to work in this new scientific (and social) environment. 


\section{References}

[1] Adolf, Marian. Clarifying mediatization. // Empedocles. 3 (2011), 2; 153-175.

[2] Deacon, David; Stanyer, James. Mediatization: key concept or conceptual bandwagon? // Media, culture \& society. 36(2014); 1032-1044.

[3] Hepp, Andreas; Hjarvard, Stig; Lundby, Knut. Mediatization: theorizing the interplay between media, culture and society. // Media, culture \& society. 37(2015); 314-324.

[4] Hoskins, Andrew. Flashbulb memories, psychology and media studies. // Memory studies. 2(2009), 2; 147-150.

[5] DC's improbable science: truth, falsehood and evidence: [blog]. http://www.dcscience.net (Access date: 2015-16-06)

[6] Koltay, Tibor; Špiranec, Sonja; Karvalics, Laszlo Z. The shift of information literacy towards research 2.0. // The journal of academic librarianship. 41(2015); 87-93.

[7] Lövgren, Daniel; Pallas, Josef. Blogging for reputation. // The governance of modern universities, 21-23 August 2013, Reykjavik, Iceland / Engwall, Lars (ed.), 2013.

[8] Lüthje, Corinna. Mediatisierte wissenschaftsinterne Kommunikation: Stand der Forschung und theoretische Rahmung. // kommunikation@gesellschaft.15(2014).

[9] Marcinkowski, Frank; Kohring, Matthias. The changing rationale of science communication: a challenge to scientific autonomy. // Journal of science communication. 13(2014), 3.

[10] Nowotny, Helga. The changing nature of public science // The public nature of science under assault. / Novotny, Helga [et al.] (ed.). Berlin ; New York : Springer, 2005, 1-27.

[11] Peters, Hans Peter. Gap between science and media revisited. // Proceedings of the National Academy of Sciences. 110(2013); 14102-14109.

[12] Peters, Hans Peter; Heinrichs, Harald; Jung, Arlena; Kallfass, Monika; Petersen, Imme. Medialization of science as a prerequisite of its legitimization and political relevance. // Communicating Science in Social Contexts / Cheng, Donghong [et al.] (ed.). Dodrecht; London : Springer Netherlands, 2008, 71-92.

[13] Rhoten, Diana; Powell, Walter.W. The frontiers of intellectual property. // Annual review of law and social science. 3(2007), 1; 345-373.

[14] Rödder, Simone; Schäfer, Mike. S. Repercussion and resistance: an empirical study on the interrelation between science and mass media. // Communications. 35(2010) 3; 249-267.

[15] Strömback, Jesper. Four phases of mediatization. // The international journal of press/politics. 13(2008), 3; 228-246.

[16] Šuljok, Adrijana; Vuković Brajdić, Marija. How the Croatian daily press presents science news. // Science \& technology studies 26(2013), 1; 92-112.

[17] Weingart, Peter. Science and the media. // Research policy. 27(1998), 8; 869-879. 